\title{
The Impact of Fullerenes on the Ordering of Polyacrylonitrile During Nanocomposites Formation
}

Adam E. Imel ${ }^{1}$ and Mark D. Dadmun ${ }^{1,2}$

${ }^{1}$ Department of Chemistry, University of Tennessee, Knoxville, Tennessee 37996, United States

${ }^{2}$ Chemical Sciences Division, Oak Ridge National Laboratory, Oak Ridge, Tennessee 37831, United States

Abstract

The production of polymer nanocomposites from solution consists of the mixing of the polymer and nanoparticle in solution and subsequent evaporation of the solvent. We examine the formation of polyacrylonitrile and $\mathrm{C}_{60}$ fullerene nanocomposites, with a focus on monitoring these two steps. The results of this study indicate that the nanoparticles are individually dispersed with the polymer chains in solution prior to deposition and in the final film. As the solution becomes more concentrated, the nanoparticles are sequestered to the outer edges of the polymer crystals, altering the detected crystal structure. The self-assembled structure of the crystalline polymer is directed by the addition of $\mathrm{C}_{60}$ and manifests itself as a peak in small-angle $\mathrm{x}$-ray scattering on a length scale of $\sim 150 \AA$. The results suggest that the non-covalent molecular interactions between $\mathrm{C}_{60}$ and polyacrylonitrile matrix are sufficiently strong to alter the selfassembled morphology of the polymer and the meso- and nanoscale structures in the nanocomposite.

\section{Introduction}

Polymer nanocomposites, PNC, are a relatively new class of materials that synergistically combine the distinctive properties of a polymer and nanoparticle. Combining these properties can result in stronger, lighter materials that may also possess exotic electrical and thermal properties. For instance, a 1 vol. \% loading of graphene sheets in a poly(methyl methacrylate) 
(PMMA) matrix produced an increase in its electrical conductivity by 5 orders of magnitude. ${ }^{1}$ Also, 0.04 wt. \% loading of $\mathrm{C}_{60}$ in a PMMA matrix increased the shear moduli, glass transition temperature and longest relaxation time. ${ }^{2}$ The increases observed in the properties of nanocomposites are a result of the large surface area per unit volume that is inherent to nanoparticles. A nanoparticle's large surface area to volume ratio is important because more of the nanoparticle's surface is available to interact with the surrounding matrix while simultaneously occupying less volume. For example, a spherical nanoparticle has a surface area to volume ratio of $\frac{3}{r}$ where $\mathrm{r}$ represents the radius of the sphere, emphasizing that the smaller the sphere the greater the amount of surface area that is available for interactions.

Carbon based nanoparticles, such as $\mathrm{C}_{60}$, single-walled nanotubes and graphene, are important additives to polymers as they can incorporate novel strength, electrical and thermal properties to a polymer. Unfortunately, they also are known to aggregate in polymer matrices, resulting in poorly dispersed nanoparticles throughout the polymer matrix and less than optimal properties. The aggregates of carbon-based nanoparticles are held together by the weak van der Waals forces that allow the nanoparticles to easily slide past one another when stresses are applied, lowering the mechanical strength of the resultant nanocomposite, as well as altering the electronic properties and decreasing the surface area to volume ratio of the additive. Thus, aggregation of nanoparticles in polymer nanocomposites have detrimental effects to the properties of the ultimate PNC making the homogeneous dispersion of the nanoparticles throughout a polymer matrix an important property to control.

Strategies that are used to improve the dispersion of nanoparticles in a polymer matrix include covalent bonding of polymers to the nanoparticle and exploitation of non-covalent interactions between the nanoparticle and polymer. Covalent attachment of a polymer chain to a 
nanoparticle that is chemically similar to the matrix occurs by a chemical reaction bonding a polymer chain to the nanoparticle or by 'growing' the polymer chain from the nanoparticles native surface. Both strategies of covalent attachment usually result in a PNC with welldispersed nanoparticles. ${ }^{3}$ Unfortunately, the covalent attachment of a molecule to the graphitic carbon nanoparticle structure disrupts the aromatic nature of the $\pi$-electrons, thus altering the electronic properties of the nanoparticles and assuredly those of the final PNC as well. ${ }^{3}$ In addition, attaching polymers onto nanoparticles produces a polymer shell around the nanoparticle that limits the interactions between the matrix and the native nanoparticle surface. On the other hand, non-covalent interactions such as the use of electron donor-acceptor interactions or van der Waals forces preserve the inherent properties of the nanoparticles. ${ }^{4}$ Thus, strategies that exploit non-covalent interactions are a preferable route to creating nanocomposites with improved dispersion, but the impact of these interactions on the polymer assembly must be understood to create polymer nanocomposites that can achieve a range of desired properties.

Preparation methods of a polymer nanocomposite can also impact the dispersion of the nanoparticle in the final nanocomposite. ${ }^{5}$ A common method to fabricate a thin film PNC with well-dispersed nanoparticles utilizing non-covalent interactions is via solution casting. The limited availability of mutually good solvents for both the nanoparticle and polymer often results in the PNC being cast from a mixed solvent solution. In this process, the nanoparticle and polymer are dissolved separately in their respective solvents and the solutions are combined to produce the PNC solution. The structures and interactions among the two solvents, the nanoparticle, and the polymer in the PNC solution will significantly affect the dispersion of the nanoparticle in this solution, and thus in this final film, and yet is an area that is not well studied. For instance, strong attractive interactions between a polymer and a 'good' solvent will produce 
a polymer conformation that will be more expanded than if these interactions are unfavorable, such as in a theta solvent. Alternatively, a polymer chain in a poorer solvent will more likely collapse, leading to separation of the polymer from the nanoparticle, and a less desirable nanoparticle dispersion throughout the final PNC film.

The complex thermodynamics of entropic packing and enthalpic interactions between polymer-particle, polymer-polymer and particle-particle during solvent evaporation of the PNC solution will direct the morphology final PNC. ${ }^{6,7}$ A polymer-particle interaction that is stronger than the polymer-polymer or particle-particle interaction can disrupt the packing of the polymer, which can deteriorate the properties of the pure polymer. Conversely, if the self-attraction between polymer-polymer and particle-particle is strong and unable to be overcome by the interfacial attraction between the polymer and particle, a poorly dispersed nanocomposite will usually result. For instance, nanoparticles such as $\mathrm{CdS}$ have been found to induce changes in copolymer morphologies of poly(styrene-b-4-vinyl pyridine) from hexagonally packed cylinders to lamellae $e^{8}$ and the addition of Au nanoparticles can shift the morphology of poly(styrene-b-2vinyl pyridine) copolymers from lamellae to bicontinuous gyroid. ${ }^{9}$

Therefore, the current investigation utilizes this perspective to examine how the introduction of $\mathrm{C}_{60}$ to a polyacrylonitrile (PAN) matrix alters the dispersion of the polymer and nanoparticle in solution and how this translates to the structural ordering of the polymer in a solution cast PNC. Examining the viscosity of the PNC solution monitors the impact of a mixedsolvent and $\mathrm{C}_{60}$ on the solvated behavior of the polymer by providing a measure of the polymer chain expansion in solution. The structures of $\mathrm{C}_{60}$ and the polymer chain in the solvent free PNC film are then examined by both small and wide-angle x-ray scattering. The scattering results indicate that the presence of small amounts of $\mathrm{C}_{60}$ in the casting solution alter the structural 
development of the PAN during the film drying process, and guide the PAN toward a novel structure.

\section{Methods / Experimental}

Chemicals. Polyacrylonitrile (PAN) was purchased from Scientific Polymer Products, Inc. with a listed weight average molecular weight $\left(\mathrm{M}_{\mathrm{w}}\right)$ and number average molecular weight $\left(\mathrm{M}_{\mathrm{n}}\right)$ of $102,700 \mathrm{~g} / \mathrm{mole}$ and $60,600 \mathrm{~g} / \mathrm{mole}$, respectively. The fullerenes, $\mathrm{C}_{60}$, were purchased from Bucky USA (99.5\% purity). Anisole (99\% purity) and 1, 2, 4-trichlorobenzene (TCB) (99\% purity) were purchased from Sigma Aldrich. HPLC grade toluene was purchased from Fischer Scientific. $\quad N$-methyl-2-pyrrolidinone (NMP) $(99.5 \%$ purity) and extra dry $o$ dichlorobenzene (ODCB) (99\% purity) were purchased from Acros Organics. All materials were used as received with no further purification

Sample Preparation. Polyacrylonitrile $(0.6000 \mathrm{~g})$ was dissolved in NMP $(15.0 \mathrm{~mL})$ at $100{ }^{\circ} \mathrm{C}$ with vigorous stirring in a 4-dram scintillation vial with an aluminum-lined lid. In separate 4-dram vials with aluminum-lined lids, the appropriate amount of fullerene was dissolved with 1-2 $\mathrm{mL}$ of anisole, o-dichlorobenzene (ODCB), trichlorobenzene (TCB) or toluene and bath sonicated for two hours. The solubility of $\mathrm{C}_{60}$ in the selected solvents is reported to be $2.8 \mathrm{mg} / \mathrm{ml}$ in toluene, $5.6 \mathrm{mg} / \mathrm{ml}$ in anisole, $8.5 \mathrm{mg} / \mathrm{ml}$ in TCB and $27 \mathrm{mg} / \mathrm{ml}$ in ODCB. Moreover, the maximum concentration of $\mathrm{C}_{60}$ used in the sample preparation is 1.5 $\mathrm{mg} / \mathrm{mL}$, which is significantly lower than the reported solubilities, ${ }^{10}$ and thus we expect that the $\mathrm{C}_{60}$ is well dispersed in these solutions, All $\mathrm{C}_{60}$ solutions were purple in color, which is a visual indication of individually dissolved $\mathrm{C}_{60}$ molecules. ${ }^{11,12}$ The $\mathrm{C}_{60}$ solutions were then added to the polymer solution drop wise with disposable glass pipets. The solvents in the nanocomposite solution were slowly evaporated down to $5 \mathrm{~mL}$ with a stream of air to aid the evaporation of the 
solvent. The concentrated, viscous solutions were transferred into a rectangular Teflon mold and placed in a vacuum oven at $100{ }^{\circ} \mathrm{C}$ and $762 \mathrm{~mm} \mathrm{Hg}$ of vacuum for 5 days to ensure that all of the solvent evaporated. The solvent free, solid nanocomposites were then compression molded at $135^{\circ} \mathrm{C}$ with a pressure of 5,000 lbs. for $5 \mathrm{~min}$ and 10,000 lbs. for $5 \mathrm{~min}$ with an aluminum mold $(1 \mathrm{~cm} \times 2 \mathrm{~cm} \times 0.05 \mathrm{~cm})$. Kapton $^{\circledR}$ sheets were used to protect the samples from the heated plates of the Carver $^{\circledR}$ hydraulic laboratory press.

Viscosity. Measurements were carried out with a Titronic Universal ${ }^{\circledR}$ automatic dilution instrument, attached to an AVS 370 pump, with a modified micro-Ubbelohde capillary viscometer, $0.64 \mathrm{~mm}$ diameter, and water-cooled submersion bath at $25{ }^{\circ} \mathrm{C}$, and all instruments are manufactured by Schott.

X-ray Scattering. Small angle x-ray scattering (SAXS) was performed on a Molecular Metrology instrument using $\mathrm{Cu} \mathrm{K \alpha}$ radiation $(\lambda=1.5418 \AA$ ) equipped with a two-dimensional position sensitive proportional detector of circular shape (radius $=2.5 \mathrm{~cm}$ ). A monochromatic $\mathrm{x}-$ ray source from the sealed $\mathrm{x}$-ray tube is focused by a pair of Kirkpatrick-Baez micro-focusing mirrors. The sample to detector distance was $1.5 \mathrm{~m}$ with the $q$ range $0.01 \AA^{-1}$ to $0.15 \AA^{-1}$. The $\mathrm{x}$ ray operating voltage was $45 \mathrm{kV}$ with a current of $0.66 \mathrm{~mA}$. The exposure time for measuring each sample was 1 hour. X-ray Diffraction (XRD) was performed on the polymer nanocomposites with a Rigaku, Inc. XRD instrument at room temperature, using monochromatic $\mathrm{Cu} \mathrm{K} \alpha$ radiation operating at $45 \mathrm{kV}$ and $40 \mathrm{~mA}$. Data were collected for $2 \theta$ ranging from 10 to 35 degrees. The peaks were deconvoluted with the program PeakFit@. The goodness of the fits for the XRD peaks was determined by comparing the statistical $\mathrm{R}^{2}$ values, choosing the value closest to one to be used in the analysis of the XRD peaks. 


\section{Results}

Table 1. The intrinsic viscosities of the nanocomposite solutions from which the PNC are cast.

Viscosity. The intrinsic viscosity of polymeric solutions measures the increase in the viscosity of the solution due to the addition of the polymer, as well as providing insight into the dissolved polymer conformation and polymer-solvent interactions. The more favorable the

\begin{tabular}{|c|c|}
\hline Sample & $\begin{array}{l}\text { Intrinsic } \\
\text { Viscosity } \\
(\mathrm{ml} / \mathrm{mg})\end{array}$ \\
\hline$P A N / N M P$ & 0.164 \\
\hline PAN/ NMP/ Anisole & 0.195 \\
\hline$P A N / N M P / O D C B$ & 0.195 \\
\hline$P A N / N M P / T C B$ & 0.2 \\
\hline PAN/ NMP/ Toluene & 0.186 \\
\hline PAN / NMP / Anisole / 0.262 wt. $\% C_{60}$ & 0.195 \\
\hline$P A N / N M P / O D C B / 0.526$ wt. $\% C_{60}$ & 0.164 \\
\hline$P A N / N M P / T C B / 0.525 w t . \% C_{60}$ & 0.176 \\
\hline PAN / NMP/ Toluene / 0.334 wt. $\% C_{60}$ & 0.182 \\
\hline
\end{tabular}
interactions between polymer and solvent, the more viscous a polymer solution will become due to the increased swelling of the polymer chains. Conversely, if the interactions of the solution are unfavorable, the polymer chain will collapse, thus lowering the viscosity of the solution. The intrinsic viscosities of each of the solutions from which the resultant nanocomposite films are cast are shown in Table 1. The flow times of the mixed solvent polymer solutions were measured using the same solvent ratio as the solutions from which the PNC films were cast.

The intrinsic viscosity, summarized in Table 1, shows that the addition of a second solvent to the PAN/NMP solution increases the viscosities of the solutions indicating an increase in the contribution of the polymer to the viscosity of the solution. The addition of $\mathrm{C}_{60}$ to the solutions slightly decreases the viscosity compared to the solutions without $\mathrm{C}_{60}$, but do not decrease the viscosity below that of PAN dissolved in the single solvent NMP.

The intrinsic viscosity of a polymer solution is directly related to the hydrodynamic volume, $V_{h}$ and the hydrodynamic radius, $R_{h}$ of the polymer coil by the equation, 


$$
[\eta]=\frac{5 N_{A} V_{h}}{2 M}
$$

where $N_{A}$ and $M$ represent Avogadro's number and the polymer molecular weight, respectively.

The hydrodynamic volume is then related to the polymer's hydrodynamic radius by

$$
V_{h}=\frac{4}{3} \pi R_{h}^{3}
$$

Figure 1 displays the hydrodynamic radius of the polymer in each nanocomposite solution and mixed solvent as derived from the viscosity measurements. The $R_{h}$ only varies between $13.9 \mathrm{~nm}$ and $14.8 \mathrm{~nm}$, illustrating that the addition of the second solvent and $\mathrm{C}_{60}$ to the solution do not detrimentally alter the polymer chain dimensions. This can be interpreted to indicate that these solutions accommodate the polymer and the nanoparticles well. Therefore, these results suggest that the

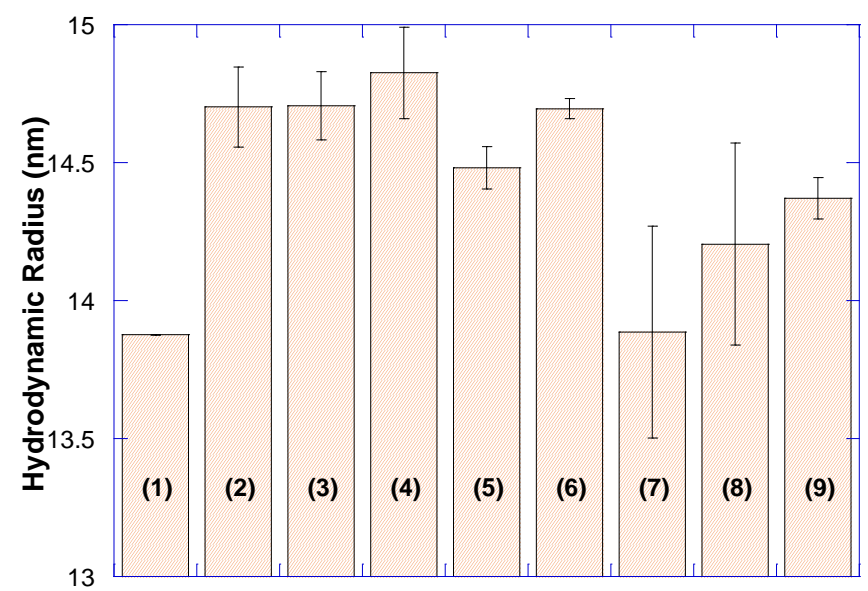
Sample

Figure 1: The hydrodynamic radius of the PAN molecule as determined from intrinsic viscosity measurements. The numbers correspond to the samples:

nanoparticles and matrix will not phase

separate during the solvent evaporation in the 1) $P A N$ in NMP

2) PAN in NMP with Anisole (15:1 solvent ratio)

3) $P A N$ in NMP with $O D C B$ (15:1 solvent ratio)

4) $P A N$ in NMP with TCB (15:1 solvent ratio)

formation of the final solvent-free film.

5) PAN in NMP with Toluene (15:2 solvent ratio)

6) PAN in NMP with 0.262 wt. $\% C_{60}$ in Anisole

7) $P A N$ in NMP with 0.526 wt. $\% C_{60}$ in $O D C B$

X-ray Diffraction. The crystalline 8) PAN in NMP with $0.525 w t . \% C_{60}$ in TCB

9) PAN in NMP with 0.334 wt. \% $C_{60}$ in Toluene

nature of atactic polyacrylonitrile (PAN) has been studied for more than 50 years, where it was originally believed that atactic PAN had little or no crystallinity. ${ }^{13-15}$ However, in an atactic polymer there exist dyads, triads and polyads of isotacticity or syndiotacticity along the polymer 
chain, i.e. chain segments that can arrange into a crystalline structure. ${ }^{13-17}$ Molecular mechanics

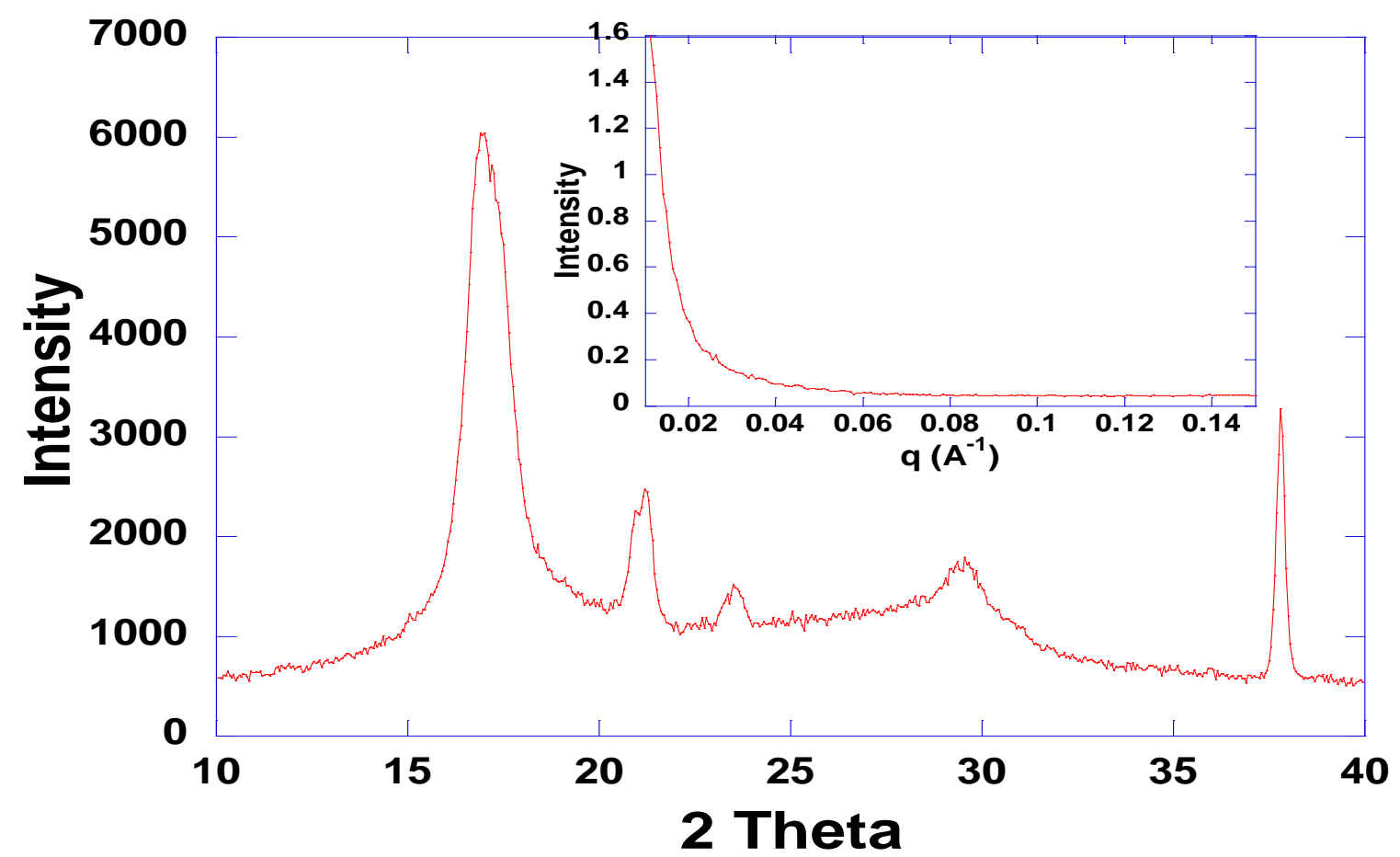

Figure 2: The XRD of the neat PAN film plotted as the intensity as a function of 2 Theta. The main peaks are at $17^{\circ}$ and $30^{\circ}$ indicating a hexagonal morphology. The middle peaks are irregular crystallites that change with the amount of isotacticity in the polymer chain. The inset is the SAXS of the neat PAN.

calculations have demonstrated that a purely isotactic PAN that is 10 monomers long will adopt a $3_{1}$ helical structure, while a pure syndiotactic PAN of the same length adopts a structure that consists of distorted zigzag conformers. A mixture of the two tacticities was shown to produce a mixture of helical and zigzag conformations. Interestingly, the isotactic portions may also adopt a conformation that emulates the planar zigzag conformations of the syndiotactic portions, thus allowing the polymer to crystallize. ${ }^{18,19}$

X-ray diffraction (XRD) experiments have examined oriented PAN fibers and the results were interpreted to show that PAN will pack into a two-dimensional ordered structure with regular chain spacing on the order of $5.2 \AA .^{13,15}$ Unfortunately, conflicting XRD and electron diffraction studies from various research groups could not unequivocally verify whether the unit cell structures of PAN are hexagonal or orthorhombic. ${ }^{20-23}$ Careful sample preparation of dry 
PAN fibers by Bashir has clarified this uncertainty by showing that solvent co-crystallization during the production of PAN fibers can induce a shift in crystalline structure of PAN from hexagonal packing of pure dry PAN to orthorhombic packing of samples that contain residual solvents. $^{20,24}$ These results show that dry PAN fibers exhibit two main XRD peaks at $17^{\circ}$ and $30^{\circ}$, which correspond to molecular distances of $5.2 \AA$ and $3.0 \AA$. This $\sqrt{3}: 1$ ratio is indicative of hexagonal packing. ${ }^{21,25-27}$ In this crystalline structure, the individual polymer chains adopt a rod-like structure that is on the order of 80 angstroms long with six PAN chains coming together producing the hexagonal polymorph. ${ }^{28}$

Alternatively, PAN fibers that form in the presence of residual solvent produce a doublet peak at $17^{\circ}$ that coincides with the co-crystallization of the solvent, which produces orthorhombic packing. ${ }^{20,21,26,29}$ As seen in Figure 2, a sample formed in our laboratory that consists of pure PAN exhibits a single peak at $17^{\circ}$ and a second peak at $30^{\circ}$, confirming hexagonal packing as the crystalline structure of this sample. The $30^{\circ}$ peak is assigned to the crystalline structure of isotactic PAN, where the isotactic polymer chain mimics the packing of a syndiotactic chain in a zig-zag fashion. ${ }^{30}$ The middle two peaks between $20^{\circ}$ and $25^{\circ}$ are assigned to small imperfect crystallites or molecular aggregates, which vary with the amount of isotacticity in the polymer chain, as well as the sample preparation method. ${ }^{16,27,29-31}$ The inset in Figure 2 shows the small angle $\mathrm{x}$-ray scattering from our pure PAN sample illustrating that there are no larger structural features in the pure PAN sample at this length scale. The single WAXS peak at $17^{\circ}$ confirms that the sample preparation used for this sample produces a PAN film that is void of any solvent.

The XRD of the nanocomposites are more complex than that of the pure PAN, where the results are summarized in Table 2 and a representative set of data is shown in Figure 3. The $17^{\circ}$ 
peak of the pure PAN sample is best fit by a single peak, while the $17^{\circ}$ peak of the nanocomposites are best modeled by two peaks centered around $17^{\circ}$. The splitting of the $17^{\circ}$ peak is a strong indicator that the polymorph shifts from hexagonally close packed to an orthorhombic packing. The $30^{\circ}$ peak, shown in Figure 2, which corresponds to the isotactic polymer chains that mimic syndiotactic packing, remains relatively unchanged for all samples.

It is important to note that the XRD of the nanocomposites do not show a peak at $11^{\circ}$, which is specific to crystalline $\mathrm{C}_{60}$, therefore the $\mathrm{C}_{60}$ does not phase separate in these samples. The presence of $\mathrm{C}_{60}$ also alters the percentage of crystallinity in the films, which is calculated from the ratio of

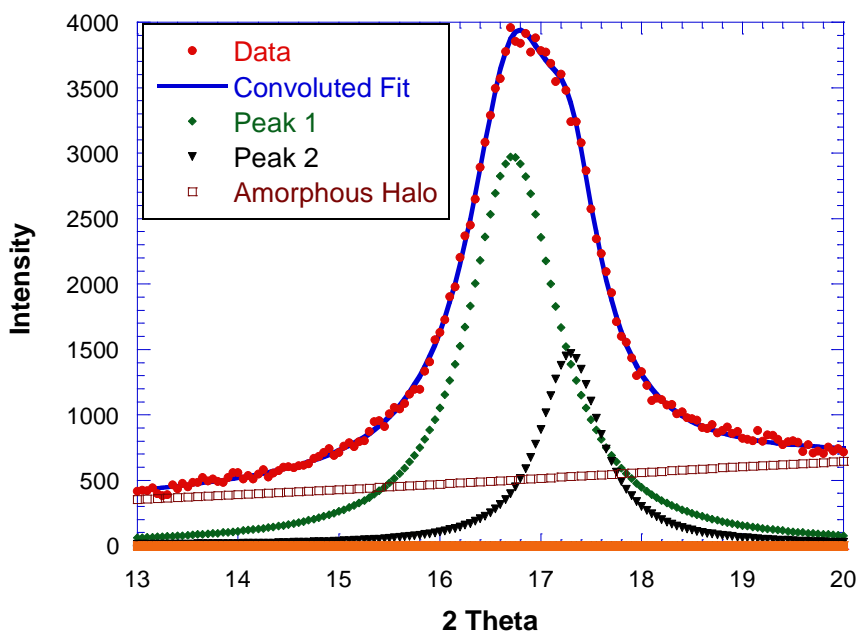
the crystalline peak area, $A_{c}$, and total area of the XRD curve,

$$
\% \text { Crystallinity }=\frac{A_{c}}{A_{T}} * 100
$$

where the total area, $A_{T}$, includes the area of the broad scattering peak attributed to amorphous content. This analysis indicates that the pure PAN film is $\sim 60 \%$ crystalline, likely due to the slow evaporation of solvent during the sample preparation, which allows more time for the polymer chains to pack into the crystalline lattice. ${ }^{30}$ As the data shows in Table 2, the addition of $\mathrm{C}_{60}$ significantly decreases the amount of crystalline polymer in the samples. The decrease in crystallinity is intriguing because the addition of nanoparticles such as $\mathrm{C}_{60}$ can act as a nucleation site for crystalline growth, which would increase polymer crystallinity. Rather, the addition of 
the nanoparticles appears to inhibit the longitudinal chain packing, which is commensurate with the consistent distribution of the $\mathrm{C}_{60}$ throughout the sample.

This may be understood by a physical picture of the crystal formation process that initially includes the $\mathrm{C}_{60}$ distributed homogeneously throughout the polymer, but leads to the exclusion of the $\mathrm{C}_{60}$ from the crystal as it grows latitudinally. This process results in the segregation of the $\mathrm{C}_{60}$ to the periphery of the long rigid crystals. This would manifest as a crystalline PAN structure that is decorated with $\mathrm{C}_{60}$ on the outside of the PAN crystalline regions. This is also consistent with the idea that the $\mathrm{C}_{60}$ acts like a residual solvent that is present during the PAN crystallization, which would explain the subtle shift from hexagonal to the orthorhombic structure.

The size of the PAN crystallites in the neat polymer and nanocomposites were also evaluated with the Scherrer equation.

$$
\tau=\frac{K \lambda}{\beta \cos (\theta)}
$$

In this equation, $\tau$ is the crystallite size in nanometers, $\mathrm{K}$ is a constant that is typically 0.9 for polymeric systems, $\lambda$ is the wavelength of radiation $(0.154 \mathrm{~nm}), \beta$ represents the full-width at half maximum (FWHM) of the XRD peaks and $\theta$ is the angle of diffraction in radians. ${ }^{22}$ Analysis of the crystallite sizes in the composites does show larger crystallites than the pure PAN samples, with an increase of about 1-3 nanometers. It is interesting that this increase in crystalline size is similar to the length scale of the crystal in the pure PAN sample plus an additional one to three $\mathrm{C}_{60}$ nanoparticles that are incorporated at the periphery of the PAN crystal. 


\section{Small-Angle X-ray Scattering: To}

understand how these smaller structural

features correlate to larger structures, we

used small-angle $\mathrm{x}$-ray scattering

(SAXS) to investigate the presence of larger structures in the PAN nanocomposites. Figure 4 shows the

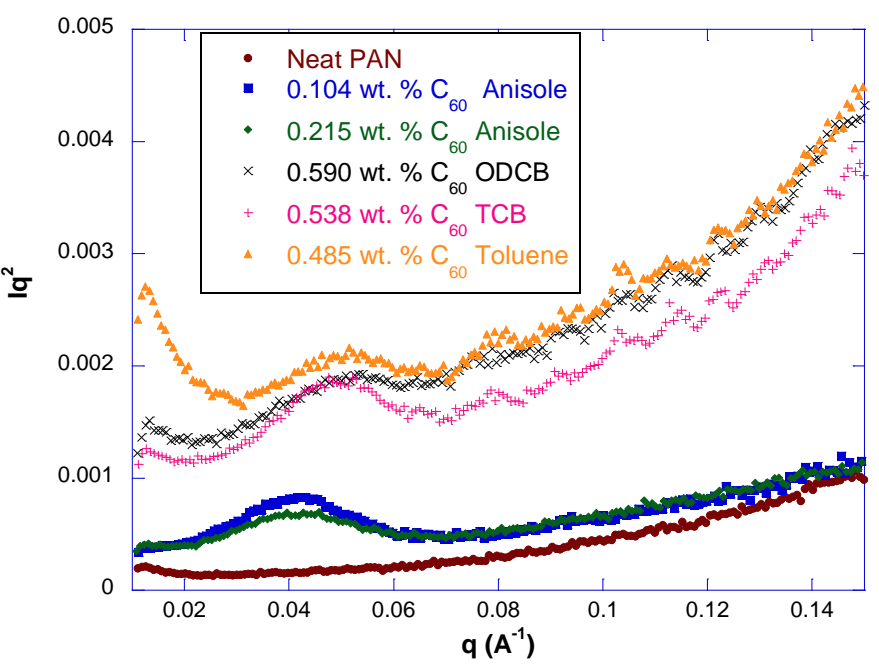
small-angle $x$-ray curves of the nanocomposites, which exhibit a peak

Figure 4: A Kratky plot of the pure PAN and PAN/C 60 nanocomposites formed from mixed solvents emphasizes the lack of features in the neat PAN and the peak that is present after the addition of $C_{60}$.

that is absent in the SAXS curve of the pure PAN sample (Fig 2 inset). The pure PAN does not feature a peak while the nanocomposites all have a peak around 0.04-0.05 $q\left(\AA^{-1}\right)$. The relationship $\frac{2 \pi}{q}=d$ relates $q$-space into the real space distance. The addition of $\mathrm{C}_{60}$, therefore, produces structures that have a dominant length scale between $157 \AA$ and $125 \AA$. The data in Figure 4 are presented as a Kratky plot to minimize the low- $q$ scattering and emphasize the features in the curves at higher $q$ in the SAXS pattern. As can be seen in Figure 4, all of the samples exhibit a positive slope towards high- $q(>0.06)$. The upward slope in the Kratky plot indicates a more rigid structure at smaller length scales. The crystals of the PAN polymer, which are the scattering object, have been previously described as rod-like, thus this result is not surprising. The persistence length, $l_{p}$, of this rod-like structure can be calculated by noting the characteristic $q$ value, denoted by $q^{*}$, at which the curve deviates from the horizontal to an upward slope, with the equation

$$
l_{p}=\frac{6}{\pi q^{*}} \cdot 32
$$


These results, summarized in Table 2, show that the persistence length of the crystals in the nanocomposites are significantly less than those of the pure polymer, implying that the rod-like PAN crystals are longer in the pure PAN than in the PAN nanocomposites. This can also be explained by the presence of the $\mathrm{C}_{60}$ nanoparticles disrupting the packing of the polymer chains into long crystals, thus decreasing the rigidity or persistence length of the rod-like crystals. Our analysis of the wide-angle scattering results implies that the $\mathrm{C}_{60}$ resides on the outside of the PAN crystalline regions, limiting the latitudinal growth of the crystals, while the SAXS data indicates that the presence of the $\mathrm{C}_{60}$ also limits the longitudinal growth of the PAN crystals. This interpretation therefore indicates that the $\mathrm{C}_{60}$ significantly limits the growth of the PAN crystals in all directions.

Directing our focus to the low- $q$ region $(<0.06)$ in the SAXS pattern (Figure 4), a broad peak is present for the SAXS data of the nanocomposites, which is absent in the SAXS curve of the pure PAN sample. A common interpretation of a SAXS peak that is similar to those shown in Figure 4 is that of a long period of the crystals, where the long period is described as the distance between two adjacent crystalline regions or mathematically as

$$
\text { long period }=\frac{1}{2} w_{c}+w_{a}+\frac{1}{2} w_{c}
$$

where $w_{c}$ is the width of crystalline area and $w_{a}$ is the width of the amorphous region.

However, the interpretation that this peak is a signature of the long period is not consistent with the XRD results. The addition of the nanoparticle decreases the amount of crystallinity, as shown in Table 2. A consequence of the decrease in crystallinity is an increase in the distance between crystalline regions, as less crystallinity results in larger amorphous regions, thus larger crystalline separation. If the peak observed in the nanocomposite SAXS 
curves were a measure of the spacing between crystalline regions, doubling the crystallinity would decrease the long period by approximately half. If this were the case, the pure PAN sample would have a peak near $12^{\circ}-10^{\circ}$ in the XRD profile, but it does not. Moreover, the pure PAN sample with the highest crystallinity does not exhibit a peak in the SAXS $q$-range, further strengthening the interpretation that this peak is not related to the crystalline long period.

\section{$\underline{\text { Discussion }}$}

The production of the $\mathrm{PAN} / \mathrm{C}_{60}$ polymer nanocomposites consists of two fundamental steps, first the mixing of the two individual solutions that contain the PAN and $\mathrm{C}_{60}$, followed by the evaporation and subsequent concentration of the combined solution until the PNC is solventfree. The combination of the two solutions may result in a significant decrease in solvent quality for the polymer or nanoparticle, and hinder the homogeneous dispersion of nanoparticles and polymer in solution, ultimately affecting the distribution of the nanoparticles in the polymer matrix in the final film. For this reason, we chose solvents for $\mathrm{C}_{60}$ that range in solubility from very good (ODCB) to marginal (Toluene) to gain insight into how the thermodynamics of a mixed solvent PNC solution will affect the dispersion of the nanoparticles in the final polymer nanocomposite. The PNC solutions consisted of polymer and fullerene dissolved in a mixed solvent with a solvent ratio of 15:1 for NMP:anisole, NMP:ODCB, and NMP:TCB and 15:2 for NMP:Toluene. The viscosity data in Table 1 and the hydrodynamic radii in Figure 1 indicate that the $\mathrm{C}_{60}$ and polymer solutions from which the nanocomposites are fabricated are a homogeneously dispersed PNC solution. During the solvent evaporation process, the swollen polymer chains will begin to self-assemble into their final morphology as the solution becomes more concentrated. The SAXS and XRD results of this study indicate that the described selfassembly process excludes the $\mathrm{C}_{60}$ nanoparticles from the crystalline PAN structures, but the 
presence of the $\mathrm{C}_{60}$ directs the structural ordering of the PAN. The XRD results provide evidence that the $\mathrm{C}_{60}$ behaves as a residual solvent and shifts the PAN crystal structure from hexagonal packing in the pure PAN to an orthorhombic morphology in the nanocomposites. The results from the viscosity and XRD experiments suggest that during film formation and prior to PAN crystallization, the $\mathrm{C}_{60}$ is homogeneously dispersed in the film. Once PAN crystallization commences, the $\mathrm{C}_{60}$ is excluded from these crystalline regions, and is thus sequestered to the outer edges of the PAN crystals. This is consistent with the shift in PAN crystalline morphology, with the $\mathrm{C}_{60}$ behaving in a similar manner to a residual solvent. The SAXS data illustrates a decrease in the persistence length of the PAN crystals in the nanocomposite, which is also consistent with the confinement of the $\mathrm{C}_{60}$ to the outer boundary of the PAN crystals. Moreover, the presence of the $\mathrm{C}_{60}$ at the edges of the PAN crystals hinders the crystalline growth significantly decreasing the percent crystallinity in the PNC.

Sequestration of the $\mathrm{C}_{60}$ to the periphery of the PAN crystals and the decrease in the percent crystallinity is also consistent with the appearance of the SAXS peak. The SAXS peak results from a distribution of rod-like PAN crystals that are approximately 8-10 nm wide surrounded by an amorphous $\mathrm{C}_{60}$ rich layer that is about $4-6 \mathrm{~nm}$ thick, producing a scattering domain that is on the order of 14-16 $\mathrm{nm}$. The PAN crystalline domain that is decorated with amorphous $\mathrm{C}_{60}$ on the periphery will appear as a single structure in x-ray scattering because these two materials have similar scattering length densities (SLD), because SLD in x-ray scattering comes from the electron density in the sample. To test this hypothesis, the scattering length densities of the PAN crystal and the $\mathrm{C}_{60}$ fullerene can be estimated. A lower-bound estimate of the $\mathrm{x}$-ray SLD for PAN is $1.10 \times 10^{-5} \AA^{-2}$, based on a density of $1.22 \mathrm{~g} / \mathrm{mL}$ for $\mathrm{PAN}^{33}$, which includes contributions from the lower density amorphous regions and higher density crystalline 
regions. If we assume a $10 \%$ increase in density for crystalline PAN, the resulting SLD of the PAN crystal is $1.21 \times 10^{-5} \AA^{-2}$. The $\mathrm{C}_{60}$ in these nanocomposites is amorphous, since the XRD does not show a crystalline peak for the fullerene. The density of crystalline $\mathrm{C}_{60}$ is reported to be $1.65 \mathrm{~g} / \mathrm{mL}^{34}$, assuming that the density of amorphous $\mathrm{C}_{60}$ is $10-20 \%$ less than that of crystalline $\mathrm{C}_{60}$ leads to an SLD of $1.26 \times 10^{-5} \AA^{-2}-1.12 \times 10^{-5} \AA^{-2}$. Thus, the SLD of the PAN crystal and amorphous $\mathrm{C}_{60}$ are quite similar and thus these two materials will appear as similar structures in $\mathrm{x}$-ray scattering. Moreover, this information is consistent with the structure of the final nanocomposite consisting of PAN crystals decorated with $\mathrm{C}_{60}$ fullerenes on their periphery, which results from the directed assembly of the PAN crystal by the $\mathrm{C}_{60}$ during film formation.

\section{Conclusion}

Viscosity measurements of PAN in a single solvent, mixed solvents, and in solution with $\mathrm{C}_{60}$ indicate the nanoparticle and polymer are homogeneously dispersed in solution prior to nanocomposite formation, which is advantageous for producing a well-dispersed PNC film. Additionally, the choice of a second solvent and $\mathrm{C}_{60}$ does not significantly alter the size of the crystals in the nanocomposite films.

The XRD of pure PAN confirms that the sample preparation does not leave residual solvent, as indicated by the formation of a hexagonally closed packed unit cell. The pure PAN sample also exhibits an upward slope at high- $q$ in a Kratky plot of the SAXS data, indicating the presence of rod-like structure of the crystals. The addition of $\mathrm{C}_{60}$ to the PAN matrix, however, results in significant changes to the SAXS and XRD scattering, which includes the appearance of a broad peak in the SAXS and a shift of crystalline packing from hexagonal to orthorhombic. The amount of PAN crystallinity in the nanocomposites is inhibited by the inclusion of $\mathrm{C}_{60}$, 
where the crystallinity of the nanocomposite is less than half that of the pure polymer. This also manifests itself as a reduction in persistence length of the rod-like PAN crystals.

A structural model that can account for the change in the scattering patterns with the addition of $\mathrm{C}_{60}$ is one where the PAN packs into rod-like crystals with hexagonal packing of the polymer chains in the neat polymer film. The addition of $\mathrm{C}_{60}$ nanoparticles to the $\mathrm{PNC}$ solution results in a homogeneous distribution of $\mathrm{C}_{60}$ in the polymer prior to crystallization, which is followed by the sequestration of the $\mathrm{C}_{60}$ to the outer edges of the PAN crystals during crystallization, producing an orthorhombic crystal morphology in the final film. The addition of $\mathrm{C}_{60}$ also inhibits the crystallization process of the PAN chains, as indicated by the decrease in percent crystallinity and decrease in persistence length. A consistent description that explains the subtle shift in crystalline morphology, the decrease in crystallinity, the decrease in persistence length, as well as the appearance of the SAXS peak is that the $\mathrm{C}_{60}$ nanoparticles envelope the rod-like PAN crystal in the final PAN/C 60 nanocomposite. Consequently, the obstruction of the PAN crystallization by the $\mathrm{C}_{60}$ in the crystallization process directs the size of crystals to a dominant length scale that manifests itself in a peak in the SAXS curves of the nanocomposites.

\section{Acknowledgement}

This research is supported by the Department of Energy, Office of Basic Energy Sciences, Division of Materials Sciences and Engineering. 


\section{The Impact of Fullerenes on the Ordering of Polyacrylonitrile During Nanocomposites Formation}

Adam E. Imel ${ }^{1}$ and Mark D. Dadmun ${ }^{1,2}$

Abstract Graphic:

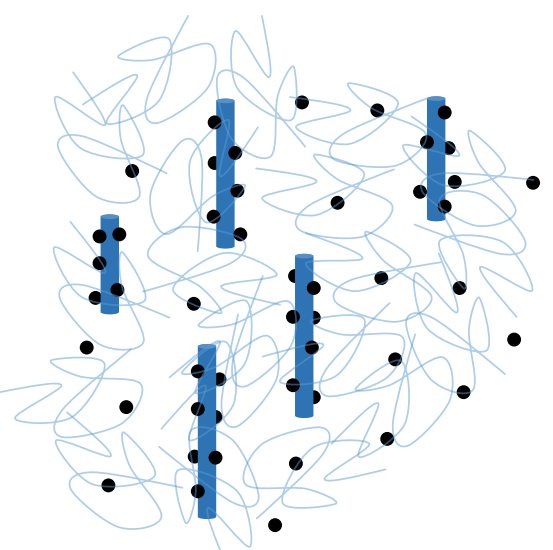


Table 2: A summary of the analyses of the XRD and SAXS curves of the neat PAN and the PAN/C 60 nanocomposites. The peak positions are presented as $2 \theta$ in degrees.

\begin{tabular}{|c|c|c|c|c|c|c|}
\hline Sample & $\begin{array}{l}\text { Peak } \\
\text { (20) }\end{array}$ & Packing & $\begin{array}{l}\text { Crystallite } \\
\text { Size (nm) }\end{array}$ & $\begin{array}{l}\text { Crystallinity } \\
(\%)\end{array}$ & $\begin{array}{l}\text { Persistence } \\
\text { Length (̊) }\end{array}$ & $\begin{array}{l}\mathbf{R}^{2} \\
\text { value }\end{array}$ \\
\hline \multirow{2}{*}{ Neat PAN } & 17.0 & \multirow{2}{*}{$\mathrm{HCP}$} & 6.27 & \multirow{2}{*}{59.6} & \multirow{2}{*}{80.7} & \multirow{2}{*}{0.985} \\
\hline & 29.5 & & 3.48 & & & \\
\hline \multirow{3}{*}{$\begin{array}{l}0.215 \text { wt. } \% \\
\mathrm{C}_{60} \text { Anisole }\end{array}$} & 16.7 & \multirow{3}{*}{ Ortho } & 9.46 & \multirow{3}{*}{24.7} & \multirow{3}{*}{34.0} & \multirow{3}{*}{0.993} \\
\hline & 17.3 & & 7.65 & & & \\
\hline & 29.3 & & 2.92 & & & \\
\hline \multirow{3}{*}{$\begin{array}{l}0.485 \text { wt. } \% \\
\mathrm{C}_{60} \text { Toluene }\end{array}$} & 16.8 & \multirow{3}{*}{ Ortho } & 9.17 & \multirow{3}{*}{15.9} & \multirow{3}{*}{29.4} & \multirow{3}{*}{0.991} \\
\hline & 17.3 & & \begin{tabular}{|l|}
10.7 \\
\end{tabular} & & & \\
\hline & 29.1 & & 3.61 & & & \\
\hline \multirow{3}{*}{$\begin{array}{l}0.538 \text { wt.\% } \\
\mathrm{C}_{60} \text { TCB }\end{array}$} & 16.7 & \multirow{3}{*}{ Ortho } & 7.38 & \multirow{3}{*}{23.9} & \multirow{3}{*}{36.9} & \multirow{3}{*}{0.986} \\
\hline & 17.3 & & 12.2 & & & \\
\hline & 29.1 & & 5.05 & & & \\
\hline \multirow{3}{*}{$\begin{array}{l}0.590 \text { wt.\% } \\
\mathrm{C}_{60} \text { ODCB }\end{array}$} & 16.6 & \multirow{3}{*}{ Ortho } & 8.18 & \multirow{3}{*}{23.7} & \multirow{3}{*}{37.9} & \multirow{3}{*}{0.992} \\
\hline & 17.2 & & 8.62 & & & \\
\hline & 29.3 & & 4.00 & & & \\
\hline
\end{tabular}




\section{References}

1. Yang, Y.-K. et al. Non-covalently modified graphene sheets by imidazolium ionic liquids for multifunctional polymer nanocomposites. J. Mater. Chem. 22, 5666 (2012).

2. Kropka, J. M., Putz, K. W., Pryamitsyn, V., Ganesan, V. \& Green, P. F. Origin of Dynamical Properties in PMMA- $\mathrm{C}_{60}$ Nanocomposites. Macromolecules 40, 5424-5432 (2007).

3. Linton, D. et al. The importance of chain connectivity in the formation of non-covalent interactions between polymers and single-walled carbon nanotubes and its impact on dispersion. Soft Matter 6, 2801 (2010).

4. Georgakilas, V. et al. Functionalization of graphene: covalent and non-covalent approaches, derivatives and applications. Chem. Rev. 112, 6156-214 (2012).

5. Lee, C.-U. \& Dadmun, M. D. Improving the dispersion and interfaces in polymer-carbon nanotube nanocomposites by sample preparation choice. J. Polym. Sci. Part B Polym. Phys. 46, 1747-1759 (2008).

6. Jancar, J. et al. Current issues in research on structure-property relationships in polymer nanocomposites. Polymer 51, 3321-3343 (2010).

7. Hooper, J. B. \& Schweizer, K. S. Real Space Structure and Scattering Patterns of Model Polymer Nanocomposites. Macromolecules 40, 6998-7008 (2007).

8. Yeh, S.-W., Wei, K.-H., Sun, Y.-S., Jeng, U.-S. \& Liang, K. S. CdS Nanoparticles Induce a Morphological Transformation of Poly(styrene- b -4-vinylpyridine) from Hexagonally Packed Cylinders to a Lamellar Structure. Macromolecules 38, 6559-6565 (2005).

9. Kim, B. J., Bang, J., Hawker, C. J. \& Kramer, E. J. Effect of Areal Chain Density on the Location of Polymer-Modified Gold Nanoparticles in a Block Copolymer Template. Macromolecules 39, 4108-4114 (2006).

10. Ruoff, R. S., Tse, D. S., Malhotra, R. \& Lorents, D. C. Solubility of C60 in a Variety of Solvents. J. Phys. Chem. 97, 3379-3383 (1993).

11. Coustel, N. et al. Purification of C60 by a simple crystallization procedure. J. Chem. Soc. Chem. Commun. 1402-1403 (1992).

12. Mirau, P. a. \& Lyons, M. Intermolecular Interactions and Dynamics in Polymer/ $\mathrm{C}_{60}$ Blends. Macromolecules 43, 625-629 (2010).

13. Holland, V. Crystalline morphology of polyacrylonitrile. J. Polym. Sci. 43, 1953-1955 (1960). 
14. Holland, V. F., Mitchell, S. B., Hunter, W. L. \& Lindenmeyer, P. H. Crystal structure and morphology of polyacrylonitrile in dilute solution. J. Polym. Sci. 62, 145-151 (1962).

15. Bohn, CR, Schaefgen, JR, Statton, W. Laterally ordered polymers: Polyacrylonitrile and poly(vinyl trifluoroacetate). J. Polym. Sci. 55, 531-549 (1961).

16. Krigbaum, W. \& Tokita, N. Melting point depression study of polyacrylonitrile. J. Polym. Sci. 43, 467-488 (1960).

17. Chiang, R. Crystallization and melting behavior of polyacrylonitrile. J. Polym. Sci. Part A Gen. Pap. 1, 2765-2775 (1963).

18. $\mathrm{Hu}, \mathrm{X}$. The molecular structure of polyacrylonitrile fibers. J. Appl. Polym. Sci. 62, 19251932 (1996).

19. Hobson, R. \& Windle, A. Crystalline structure of atactic polyacrylonitrile. Macromolecules 26, 6903-6907 (1993).

20. Bashir, Z. Co-crystallization of solvents with polymers: The x-ray diffraction behavior of solvent-containing and solvent-free polyacrylonitrile. J. Polym. Sci. Part B Polym. Phys. 32, 1115-1128 (1994).

21. Atureliya, S. K. \& Bashir, Z. Continuous plasticized melt-extrusion of polyacrylonitrile homopolymer. Polymer 34, 5116-5122 (1993).

22. Tan, L., Chen, H., Pan, D. \& Pan, N. Investigation into the gelation and crystallization of polyacrylonitrile. Eur. Polym. J. 45, 1617-1624 (2009).

23. Bashir, Z. \& Rastogi, S. The Explanation of the Increase in Slope at the Tg in the Plot of d-Spacing Versus Temperature in Polyacrylonitrile. J. Macromol. Sci. Part B 44, 55-78 (2005).

24. Bashir, Z. The hexagonal mesophase in atactic polyacrylonitrile: A new interpretation of the phase transitions in the polymer. J. Macromol. Sci. Part B 40, 37-41 (2001).

25. Ge, H., Liu, H., Chen, J. \& Wang, C. The microstructure of polyacrylonitrile-stabilized fibers. J. Appl. Polym. Sci. 113, 2413-2417 (2009).

26. Bashir, Z. The effect of solvent on the X-ray scattering from polyacrylonitrile. J. Mater. Sci. Lett. 12, 1526-1528 (1993).

27. Bashir, Z. Thermoreversible Gels of Polyacrylonitrile. J. Polym. Sci. Part B Polym. Phys. 30, 1299-1304 (1992).

28. Bashir, Z. A critical review of the stabilisation of polyacrylonitrile. Carbon 29, 10811090 (1991). 
29. Bashir, Z.,Atureliya,SK, Church, S. Production of oriented polyacrylonitrile and crystallization from solution. J. Mater. Sci. 28, 2721-2732 (1993).

30. Minagawa, M., Taira, T., Yabuta, Y., Nozaki, K. \& Yoshii, F. An Anomalous Tacticity-Crystallinity Relationship: A WAXD Study of Stereoregular Isotactic (83-25) Polyacrylonitrile Powder Prepared by Urea Clathrate Polymerization. Macromolecules 34, 3679-3683 (2001).

31. Allen, R. a., Ward, I. M. \& Bashir, Z. The variation of the d-spacings with stress in the hexagonal polymorph of polyacrylonitrile. Polymer 35, 4035-4040 (1994).

32. Donatti, D. A., Awano, C. M., de Vicente, F. S., Ruiz, A. I. \& Vollet, D. R. Persistence Length, Mass Fractal, and Branching in the Aggregating of Vinyltriethoxysilane-Derived Organic/Silica Hybrids. J. Phys. Chem. C 115, 667-671 (2011).

33. Hurley, R. B. \& Tzentis, L. S. Density of polyacrylonitrile. J. Polym. Sci. Part B Polym. Lett. 1, 423-426 (1963).

34. Thornton, T. \& Smith, D. Density of fullerene containing soot as determined by helium pycnometry. Chem. Phys. Lett. 186, 456-458 (1991). 